\title{
LUCRO, EMPRESA Y RELIGIÓN: EL CASO HOBBY LOBBY
}

\author{
PROFIT, BUSINESS CORPORATIONS AND RELIGION: \\ THE HOBBY LOBBY CASE
}

\author{
Luis Alejandro Silva IrarráZaval ${ }^{1}$
}

\begin{abstract}
RESUMEN: La Corte Suprema de los Estados Unidos declaró, en la sentencia Burwell v. Hobby Lobby, que las empresas comerciales están legitimadas para reclamar el derecho a la libertad religiosa. Con ello, la Corte precisó la correcta interpretación de la Religious Freedom Restoration Act. También aclaró que las empresas comerciales cumplen una finalidad más amplia que la sola maximización de utilidades. Y, por último, reconoció que la religión y los negocios son compatibles. En este artículo se analizan los argumentos de la discusión que rodeó al caso Hobby Lobby. Aparte del interés que este caso representa en sí mismo, es interesante para Chile, en consideración a los eventuales escenarios de conflicto entre personas jurídicas, con y sin fines de lucro, y las políticas del Gobierno.
\end{abstract}

Palabras clave: Libertad religiosa, legitimidad activa, Corte Suprema de los Estados Unidos, lucro.

ABSTRACT: For-profit corporations have legal standing to demand legal protection under the Religious Freedom Restoration Act (RFRA). In Burwell v. Hobby Lobby, the US Supreme Court granted RFRA protection to a business entity, thereby exempting it from a rule of general applicability. It did so on the grounds that such organizations are comprised by the word person mentioned in RFRA. But also because business corporations may pursuit ends other than profit maximization. And finally, it ruled against the federal Government because commercial and religious spheres are not to be regarded as if they were incompatible. Besides the interest this decision has in and of itself, it is worth discussing it for the importance it may have to Chile. Since chances of conflict between the Government and corporations, either nonprofit or for-profit, increase over time, the Hobby Lobby case represents a source for useful and tested arguments for the local debate.

Key word: Religious freedom, legal standing, US Supreme Court, profit.

\section{INTRODUCCIÓN}

El caso Burwell v. Hobby Lobby ${ }^{2}$, decidido por la Corte Suprema de los Estados Unidos el 30 de junio de 2014, planteó la cuestión de si una persona jurídica con fines de lucro podía alegar el derecho a la libertad religiosa. La sentencia declaró que sí podía, resolviendo

\footnotetext{
1 Profesor de Derecho Constitucional, Facultad de Derecho de la Universidad de los Andes. Mons. Alvaro del Portillo 12455, Las Condes, Santiago, Chile. Email: 1silva@uandes.cl. El presente trabajo es fruto de mi estadía de investigación en Princeton University como 2013-14 William E. Simon Visiting Fellow in Religion and Public Life del James Madison Program.

2573 U.S. _ (2014). Puesto que la publicación de la sentencia en el United States Reports TODAVÍA NO HA SIDO PUBLICADA, Y PROBABLEMENTE FALTEN UN PAR DE AÑOS (EL ÚLTIMO TOMO PUBLICADO ES EL N ${ }^{\circ} 554$ Y CORRESPONDE AL PERIODO 2012) está pendiente, no es posible incluir la página en la cita del caso. Por la misma razón, cada vez que citamos algún texto de la sentencia,
} 
así una discusión que tenía enfrentado al Gobierno de Barack Obama con el mundo conservador del país del norte. La decisión Hobby Lobby ha llevado un paso más adelante la protección de la libertad religiosa, afirmando que la actividad comercial es compatible con el ejercicio de la religión, y que el hecho de organizarse como empresa no estrecha el ámbito para reclamar del Estado respeto por las personales convicciones de fe.

El precedente establecido en el caso Hobby Lobby es de interés para Chile, porque de un tiempo a esta parte, son varios los signos que indican un eventual aumento de los conflictos entre las políticas del Gobierno y las convicciones religiosas proyectadas a través de personas jurídicas. Un ejemplo relativamente reciente es el debate habido entre el Rector de la Universidad Católica de Chile y el Gobierno de Michelle Bachelet. Cuando este último anunció el eventual envío de un proyecto de ley de aborto $^{3}$, el rector de la Universidad Católica declaró que en la red de salud de la Universidad el aborto no se practicaría "aunque la ley lo mande". Su postura se apoya en la primacía que tiene seguir la propia conciencia antes que la ley: "[a]ntes de las leyes están las convicciones más profundas de respeto a la vida, y por lo tanto he sido bien claro en que una cosa es lo que diga la ley y otra cosa es lo que a nosotros, como católicos y convencidos de esta situación, se nos vaya a obligar a hacer" ${ }^{3}$. ¿Puede extenderse la conciencia individual a la esfera de una persona jurídica? ¿Puede decirse que una persona jurídica tiene conciencia, y en qué sentido? ¿Podría eximirse una persona jurídica del cumplimiento de la ley alegando objeción de conciencia? La respuesta a estas y otras preguntas pueden aprovecharse de lo discutido y resuelto en el caso Hobby Lobby ${ }^{5}$.

Un caso anterior que demuestra aún más claramente el interés del precedente sentado por el caso Hobby Lobby es el conflicto que se produjo entre el primer Gobierno de Michelle Bachelet (2006-2010) y tres cadenas de farmacias que se negaban a comercializar la píldora del día después ${ }^{6}$. Este caso es particularmente ilustrativo porque las farmacias fueron finalmente forzadas a vender la droga en cuestión pese a las objeciones de conciencia de sus dueños o controladores, porque negarse significaba la quiebra económica del negocio ${ }^{7}$. La cuestión que se plantea en el caso de las farmacias es más complejo que en el de la Universidad Católica y el aborto, porque, además de las preguntas que arriba se plantearon, surge una arista nueva: ¿podrá una empresa comercial alegar objeción de conciencia para reclamar del Gobierno una excepción a las leyes de aplicación general?

\footnotetext{
hacemos referencia a la página de la decisión en la versión disponible en http://www.supremecourt.gov/ opinions/13pdf/13-354_olp1.pdf [consultada 24 de enero de 2015].

3 Discurso presidencial 21 de mayo de 2014, disponible en http://www.21demayo.gob.cl/pdf/2014_discurso21-mayo.pdf, p. 28.

4 http://www.emol.com/noticias/nacional/2014/05/23/661757/rector-de-la-uc-y-proyecto-de-aborto-hay-queponer-sobre-la-mesa-los-derechos-del-que-esta-por-nacer.html [consultada 13 de diciembre de 2014].

5 La analogía entre este caso y Hobby Lobby está limitada por la diferencia entre la naturaleza de la Pontificia Universidad Católica de Chile y la cadena de tiendas americana: la primera no tiene fines de lucro y la segunda sí. No obstante, la comparación puede justificarse porque en el debate jurídico sobre el caso Hobby Lobby, hay un argumento que relativiza la importancia de la distinción lucro/no lucro. Ver sección 3.3.

6 El Mercurio, Domingo 14 de octubre de 2007, p. A1.

7 El Mercurio, Domingo 28 de octubre, pp. B4 y B5.
} 
En el presente trabajo se ofrece un análisis del debate provocado por el caso Hobby Lobby respecto de la posibilidad de que personas jurídicas comerciales puedan invocar la libertad religiosa para obtener del Estado una excepción a las normas de aplicación general. Conviene señalar que este análisis se centra en el problema de la legitimidad activa de las empresas, es decir, en el derecho que tendrían de acudir a los tribunales de justicia para que se defienda su libertad religiosa. En la primera parte del trabajo se describen los antecedentes del caso, sus circunstancias y su itinerario judicial. En la segunda parte se abordan cinco argumentos involucrados, directa o indirectamente, en la discusión, con referencias a otros casos análogos a Hobby Lobby. La tercera parte comprende el análisis de la sentencia Burwell v. Hobby Lobby.

\section{LOS ANTECEDENTES DEL CASO HOBBY LOBBY}

El caso Hobby Lobby es uno entre los más de 100 juicios entablados por corporaciones y empresas en contra del Gobierno de Barack Obama ${ }^{8}$. La causa de pedir en todos ellos es la misma: una excepción a la obligación de pagar a sus empleadas por anticonceptivos que pueden tener efectos abortivos ${ }^{9}$. El origen de estos juicios se remonta a la decisión del Gobierno Federal de incluir entre las prestaciones de salud básicas que debían ser cubiertas por los seguros de salud la contracepción de emergencia ${ }^{10}$. Puesto que lo común es que sean las mismas empresas o corporaciones las que aseguran la salud de sus empleados, esta obligación recaía directamente sobre ellas.

Hobby Lobby Stores Inc. es una cadena de tiendas que vende artículos de hobby. Tiene más de 600 locales repartidos en el país, emplea directamente a 23.000 personas y genera ingresos por 3.3 billones de dólares ${ }^{11}$. Es una empresa familiar fundada en 1972 por David Green y su mujer, Barbara. Los hijos del matrimonio participan activamente de su administración. La familia Green, evangélica, es profundamente religiosa y conduce sus empresas de acuerdo con sus convicciones. Así, por ejemplo, todas las tiendas Hobby Lobby cierran

\footnotetext{
${ }^{8}$ http://www.becketfund.org/hhsinformationcentral/ [consultada 13 de diciembre de 2014].

9 Aunque la causa de pedir es la misma, la extensión de lo pedido varía. En algunos casos, como Hobby Lobby, la excepción se pidió respecto de cuatro contraceptivos de emergencia, mientras que en otros, como Conestoga Wood Specialties Corp. v. Sec'y of U.S. Dep't of Health \& Human Servs, 724 F.3d 377 (3d Cir. 2013), la excepción es respecto de dos. También aquí conviene señalar que hay otros juicios contra el Gobierno Federal en que se reclama una excepción respecto de todos los anticonceptivos, independientemente de su eventual efecto abortivo, como por ejemplo Little Sisters of the Poor v. Burwell. Este caso está por ser decidido por la Corte de Apelaciones del $10^{\circ}$ Circuito.

10 Esta disposición se enmarca en la reforma al sistema de salud impulsado por el Presidente Obama, conocida popularmente como ObamaCare. La ley de 2010, llamada Affordable Care Act (ACA), delegó en el Ministerio de Salud (U.S. Department of Health and Human Services) la tarea de precisar los cuidados preventivos básicos de salud de la mujer que deberían estar cubiertos por el seguro de salud. El Ministerio le encargó la tarea a una agencia dependiente, la Health Resources and Services Administration (HRSA), quien sugirió incluir todos los anticonceptivos aprobados por la Food and Drug Adminsitration (FDA). El Ministerio de Salud hizo suya la sugerencia y la implementó a través de un acto administrativo conocido como HHS Mandate. El texto en https:// www.federalregister.gov/articles/2013/07/02/2013-15866/coverage-of-certain-preventive-services-under-the-affordable-care-act [consultada 24 de enero de 2015].

${ }_{11}$ http://www.forbes.com/companies/hobby-lobby-stores/ [consultada 13 de diciembre de 2014].
} 
los domingos porque ese es el día del Señor; la cadena cuenta con tres ministros para atender a las necesidades espirituales de sus empleados; la empresa ha donado cuantiosas sumas a iniciativas educativas de inspiración religiosa; con frecuencia financia insertos en los periódicos en los que glorifica a $\operatorname{Dios}^{12}$. En su sitio web declara que es "por la gracia y providencia de Dios que Hobby Lobby ha permanecido en el tiempo", y que la compañía está comprometida a "honrar al Señor en todo lo que hacemos, administrándose de manera consistente con las enseñanzas de la Biblia"13.

La anticoncepción de emergencia es contraria a las creencias religiosas de la familia Green. En la medida en que dichos dispositivos pueden actuar impidiendo la anidación del cigoto, se entienden como abortivos. Y el aborto es contrario a su creencia religiosa de que la vida comienza con la concepción ${ }^{14}$. Luego, la obligación impuesta por el HHS Mandate de financiar para sus empleadas contraceptivos potencialmente abortivos les impide practicar libremente su religión y vivir de acuerdo con su conciencia. Para los Green, desobedecer la ley era una posibilidad inviable puesto que la sanción por el incumplimiento era tal que, en un año, Hobby Lobby habría tenido que pagar cerca de 475 millones de dólares en multas $(1.3 \text { millones al día })^{15}$.

Enfrentado a la alternativa de violar la propia conciencia religiosa o pagar multas que habrían significado la quiebra de la empresa, Hobby Lobby decidió demandar judicialmente una excepción al HHS Mandate, invocando la Constitución y la Religious Freedom Restoration Act (RFRA) ${ }^{16}$. Para la corte de distrito del Estado de Oklahoma que primero tramitó la acción interpuesta por Hobby Lobby, no era novedoso que se invocara la libertad religiosa para obtener una excepción a la regla de aplicación general: hacía tiempo que este era un precedente en la jurisprudencia ${ }^{17}$. Tampoco era novedoso que la excepción la pidiera una persona jurídica, porque también había precedente en la materia ${ }^{18}$. En cambio, la circunstancia de ser el demandante una empresa comercial resultaba completamente novedosa;

12 Burwell v. Hobby Lobby, 573 U.S. _ (2014) p. 14

13 http://www.hobbylobby.com/our_company/ [consultada 13 de diciembre de 2014]. Esta traducción y todas las que siguen son nuestras.

14 Burwell v. Hobby Lobby, p. 14

15 Burwell v. Hobby Lobby, p. 32

16 Específicamente, se invocó la cláusula segunda - the Free Exercise Clause- de la Religious Clauses de la Primera Enmienda de la Constitución, que dispone que el Congreso no podrá dictar ley alguna prohibiendo el ejercicio de la religión. El estándar de esta prohibición fue fijado por la sentencia Employment Division, Department of Human Resources of Oregon vs. Smith, 494 U.S. 872 (1990) y consiste en que no proceden excepciones por motivos religiosos cuando se trata de leyes religiosamente neutrales y de aplicación general. RFRA, promulgada en 1993, representa la voluntad del Congreso por recuperar el estándar de la Free Exercise Clause anterior a la sentencia Smith, que era menos exigente. RFRA (42 U.S.C. ch. 21B $\$ 2000$ bb y ss.) dispone que el Gobierno no podrá gravar substancialmente el ejercicio de la religión, incluso si se trata de leyes neutras de aplicación general, a menos que se cumplan dos condiciones copulativas: que el Gobierno tenga un interés imperioso (compelling interest) en obtener un determinado resultado, y que lo haga a través del medio menos gravoso posible. El debate, en definitiva, se centró en la interpretación de RFRA. Era inevitable que esto fuera así, puesto que RFRA establece un umbral más ventajoso para el demandante que el constitucional.

17 Sherbert v. Verner, 374 U.S. 398 (1963) abrió la línea de las excepciones religiosas que, después de la decisión Smith, continuó RFRA.

18 Church of the Lukumi Babalu Aye, Inc. v. Hialeah, 508 U.S. 520 (1993). 
nunca antes una persona jurídica con fines de lucro había invocado la libertad religiosa para eximirse de la aplicación de la ley.

La corte de distrito rechazó la demanda de Hobby Lobby fundada en el hecho de que las empresas comerciales están esencialmente impedidas de practicar la religión. Puesto que, estrictamente hablando, no pueden practicar la religión, mal podrían gozar de libertad para practicarla, razonó la corte. Concluyó entonces que, careciendo radicalmente de la capacidad para ejercer una libertad, era un sinsentido reclamar su protección ${ }^{19}$. La Corte de Apelaciones del $10^{\circ}$ Circuito revocó la sentencia de primera instancia y acogió la demanda de Hobby Lobby ${ }^{20}$. Para el tribunal de alzada, la distinción entre persona jurídica y persona natural era irrelevante desde la perspectiva de la fe: en una empresa familiar como Hobby Lobby la religión de los dueños era inseparable de la persona jurídica ${ }^{21}$. El Gobierno Federal elevó una solicitud de certiorari a la Corte Suprema pidiéndole declarar que la libertad religiosa de las empresas comerciales está excluida de la protección de RFRA y de la Constitución. Pero la Corte Suprema confirmó el fallo de apelación declarando que las empresas son un medio legítimo para expresar las convicciones religiosas y que, por lo tanto, proteger la libertad religiosa de la empresa es lo mismo que proteger la libertad religiosa de los dueños ${ }^{22}$.

\section{EMPRESA, LUCRO Y RELIGIÓN: LOS ARGUMENTOS EN DEBATE}

En esta sección se analizan cinco argumentos que han estado presentes en la discusión acerca del derecho de las empresas comerciales a la libertad religiosa. El conjunto de estos argumentos abarca un ámbito más extenso que el comprendido en el caso Hobby Lobby. Sin embargo, se ha estimado conveniente incluirlos en el trabajo a fin de enriquecer la comprensión del problema y sus implicancias. Hay dos argumentos que podrían llamarse principales y tres secundarios. El primero (3.1) discurre sobre la posibilidad ontológica de que una persona jurídica pueda alegar el derecho a la libertad religiosa. Si este tipo de persona es un ente ficticio, ¿cómo podrá invocar un derecho que supone atributos esencialmente humanos? El segundo argumento (3.2) gira en torno al carácter comercial de la organización como una circunstancia que la inhabilita para reclamar libertad religiosa. La cuestión se plantea, al menos aparentemente, como una alternativa: o uno se dedica a los negocios o a la religión. Junto a los dos argumentos principales recién mencionados, otros argumentos secundarios han estado presentes en la discusión acerca de la legitimidad de las corporaciones con fines de lucro y su derecho a la libertad religiosa. El primero de estos (3.3) alerta sobre la amenaza que importa la extensión de RFRA a las empresas comerciales respecto de dos distinciones que serían legalmente relevantes. Una es la distinción lucro/

19870 F. Supp. 2d 1278 (WD Okla. 2012)

20 El objeto perseguido por Hobby Lobby en este juicio era obtener una preliminary injuction (algo así como una medida de no innovar), para no cumplir con el HHS Mandate mientras estuviera pendiente la discusión de fondo.

21 La Corte de Apelaciones consideró que la empresa Hobby Lobby Inc. tenía derecho a la protección de RFRA porque sus convicciones religiosas - las de la empresa, no la de los dueños- eran sinceras.

22 Burwell v. Hobby Lobby Inc., p. 18. Con esta decisión, la Corte Suprema sentó el precedente que zanja la división que la aplicación del HHS Mandate había producido en la jurisprudencia. Sobre esta división, KARTCHNER (2014). 
no-lucro (profit/non-profit), y la otra es la distinción secular/religioso. Este argumento advierte que una distinción poco clara entre estas categorías impactaría negativamente en dos ámbitos donde estas categorías son relevantes, a saber, impuestos federales y excepciones por motivos religiosos. El siguiente argumento (3.4) se refiere a la potencial vulnerabilidad en que se encontrarían las corporaciones religiosas como consecuencia de extender las excepciones a las corporaciones comerciales: mientras más se amplía la cobertura de RFRA, menor es la intensidad de su protección. El tercero de estos argumentos secundarios (3.5) apunta al riesgoso aumento de la demanda por excepciones religiosas que se seguiría como efecto de una interpretación demasiado flexible de RFRA. A continuación se desarrollarán cada uno de los cinco argumentos, presentando las dos caras de cada uno.

\subsection{LAS PERSONAS JURÍdicAS ¿CARECEN DE LEGITIMIDAD PARA ALEGAR LIBERTAD RELIGIOSA?}

La primera gran objeción contra la libertad religiosa de las sociedades comerciales apunta a su naturaleza jurídica, es decir, al hecho de tratarse de entes de razón distintos de las personas naturales que les dan origen. Podría formularse así: porque son personas jurídicas, están esencialmente incapacitadas para alegar libertad religiosa, y privan a sus miembros, i.e. dueños y administradores, del derecho para hacerlo. Esta objeción descansa en dos supuestos. El primero (3.1.1) es que las personas jurídicas están esencialmente incapacitadas para practicar la religión y, por lo tanto, carecen de legitimidad para reclamar su protección. El segundo (3.1.2) es que la separación que existe entre la persona jurídica y las personas naturales que actúan a través de ella es infranqueable.

\subsubsection{Personas jurídicas y práctica de la religión.}

La corte de distrito que rechazó la demanda de Hobby Lobby dijo: "[La empresas comerciales] no rezan, no pueden alabar a Dios, no pueden observar los sacramentos ni, en fin, realizar ninguna otra acción de inspiración religiosa de manera independiente y separada de la intención y dirección de los individuos que actúan en su nombre”23. En otras palabras, la práctica de la religión es exclusiva de los seres humanos ${ }^{24}$. Por lo tanto, las personas jurídicas, que no son seres humanos sino artificiales, están esencialmente impedidas de practicar la religión ${ }^{25}$. Y si no pueden practicar la religión, es un sinsentido atribuirles libertad religiosa. Esta posición enfatiza el carácter ficticio de las personas jurídicas y, consiguientemente, la radical dependencia que tienen de la ley para el reconocimiento de sus derechos ${ }^{26}$.

Como respuesta a lo anterior, cabría señalar, en primer lugar, los numerosos casos en que se les ha reconocido a las personas jurídicas, con y sin fines de lucro, atributos típica-

\footnotetext{
${ }^{23}$ p. 18. La cita corresponde a la página de la versión disponible en http://www.becketfund.org/wp-content/ uploads/2012/11/Order-for-HL-PI.pdf [consultada 24 de enero de 2014].

24 Nelson (2013) reconoce que la identidad moral de los individuos puede extenderse a ciertas asociaciones de las que forman parte, pero que entre estas no se cuentan las empresas comerciales.

25 Como afirma el título del trabajo de Rutledge (2014), "las empresas no tienen alma”.

26 Woytash (1978) advirtiendo de la necesidad de dejar de tratar a las personas jurídicas como si fueran seres humanos.
} 
mente humanos ${ }^{27}$. El hecho de que las personas jurídicas no puedan, propiamente hablando, heredar o expresarse, no ha sido impedimento para reconocerles, por ejemplo, el derecho de herencia, la libertad de prensa, o la libertad de expresión política ${ }^{28}$. En segundo lugar, habría que señalar aquellos casos en que la Corte Suprema le ha reconocido a las corporaciones religiosas el derecho a la libertad religiosa ${ }^{29}$. El hecho de que las iglesias, en cuanto personas jurídicas, no puedan rezar o alabar a Dios, no ha obstaculizado el reconocimiento de su legitimidad para invocar la protección constitucional y legal de la libertad religiosa.

\subsubsection{El velo corporativo ¿es infranqueable?}

El segundo supuesto se complementa con el anterior al afirmar que la distinción entre persona jurídica y persona natural no puede ser ignorada ${ }^{30}$. La religión de los dueños no se puede confundir con la religión de la empresa, así como no se confunden, por ejemplo, los patrimonios, la contabilidad, la responsabilidad, ni la representación ${ }^{31}$. Así como organizarse en persona jurídica tiene ventajas, también tiene desventajas. En palabras de la Corte de Apelaciones del $3^{\text {er }}$ Circuito: "La familia Hahn optó por constituirse como sociedad comercial (...) asumiendo así las ventajas y las desventajas de adoptar la forma corporativa. Simplemente no podemos ignorar la distinción entre los Hahn y [su empresa] Conestoga. Sostenemos, por tanto, que la demanda de libertad religiosa interpuesta por los dueños de la compañía no puede pasar a través del velo corporativo"32.

La estrategia del Gobierno federal para enfrentar las demandas de las empresas comerciales fue, por una parte, enfatizar que el HHS Mandate obligaba a la persona jurídica y no a la persona natural, y, por otra, que la separación conceptual entre persona jurídica y sus dueños y administradores era infranqueable. De este modo, era inviable para las personas naturales demandar con éxito la protección de su libertad religiosa. Si a esto se le agrega la premisa de que las personas jurídicas carecen esencialmente de libertad religiosa, el $H H S$

27 El catálogo de los derechos fundamentales (Bill of Rights) ha ido ampliándose para las personas jurídicas, al tiempo que se han ido debilitando los fundamentos teóricos en que se apoya esta tendencia. MAYER (1990) ofrece una mirada crítica a este fenómeno.

28 Miller (2011) ofrece un completo panorama de los derechos fundamentales atribuidos por la Corte Suprema a las personas jurídicas. El Derecho, explotando la ficción de las personas jurídicas, ha ido ampliando el catálogo de los derechos conforme a las necesidades que van planteando los siempre nuevos escenarios sociales.

29 Es la línea de precedente establecida por Church of the Lukumi Babalu Aye, Inc. v. Hialeah, 508 U.S. 520 (1993), Gonzales v. O Centro Espirita Beneficente Uniao do Vegetal, 546 U.S. 418 (2006), y Hosanna-Tabor Evangelical Lutheran Church and School v. Equal Employment Opportunity Commission, 565 U.S.

En el caso Consetoga, la Corte hace ver que esta línea de precedente no es determinante en el caso de empresas comerciales. ("El que las iglesias -como medio a través del cual los individuos practican su religión- hayan disfrutado desde hace tiempo de la protección de su libertad religiosa, no determina la cuestión acerca de si empresas comerciales y seculares debieran gozar de la misma protección.”)

30 Esta fue la opinión la Corte de Apelaciones del $3^{\text {er }}$ Circuito, Conestoga Wood Specialties Corp. v. Secy of U.S. Dep't of Health \& Human Servs., 724 F.3d 377, 388 (3d Cir. 2013). Ver nota 30.

31 Ver referencias en Willis (2013) notas al pie 178 y ss.

32 Conestoga Wood Specialties Corp. v. Sec'y of U.S. Dep't of Health \& Human Servs., 724 F.3d 377, 388 (3d Cir. 2013). 
Mandate quedaba virtualmente blindado ante cualquier objeción fundada en RFRA o en la Primera Enmienda de la Constitución ${ }^{33}$.

A este respecto, cabe señalar que, si bien la diferencia entre la persona jurídica y la(s) persona(s) natural(es) que actúa(n) a través de ella es esencial para el Derecho Corporativo, no es absoluta ${ }^{34}$. El mismo Derecho Corporativo conoce excepciones a este principio, conforme a las necesidades planteadas en dos situaciones, por lo menos. Por una parte, están las excepciones que nacen de la identidad entre los dueños y los administradores de una sociedad, es decir, aquellos casos en que la voluntad de la persona jurídica refleja más o menos exactamente la voluntad de los dueños y administradores ${ }^{35}$. El Derecho no es ciego a la diferencia que existe entre sociedades de este tipo y otras en que la propiedad está disuelta entre cientos o miles de personas, y en que los administradores son empleados contratados por los representantes de los dueños ${ }^{36}$. Por otra parte, están las excepciones que nacen de la necesidad de evitar los fraudes, abusos y malas prácticas que se aprovechan, precisamente, de la separación que media entre la persona jurídica y la persona natural ${ }^{37}$. Quizá el mejor ejemplo sea la doctrina del levantamiento del velo ${ }^{38}$.

En síntesis, la cuestión acerca de la legitimidad de las empresas para invocar el derecho a la libertad religiosa no puede resolverse recurriendo simplemente a la naturaleza artificial de las personas jurídicas ni a la separación conceptual que media entre estas y las personas naturales que las constituyen, porque en ambos casos el Derecho conoce excepciones. Por lo tanto, se trata de averiguar si, en el preciso caso del HHS Mandate, se verifica una de estas excepciones o no. El Gobierno alega que no, señalando el carácter comercial como el factor que impediría a las empresas beneficiarse de una excepción. Las empresas, por su parte, se defienden argumentando que la práctica de la religión no es incompatible con hacer negocios. En la sección que sigue se analiza el punto.

\footnotetext{
33 Así, la objeción religiosa al HHS Mandate vendría a ser no-justiciable. Referencias en HaLl y MEANs (2014), nota al pie número 10 .

34 WiLlis (2013) pp. 21 y ss.

35 Típico es el caso de las close corporations, que el Black's Law Dictionary define como "A corporation whose stock is not freely traded and is held by only a few shareholders (often within the same family)". Es una definición amplia con amplias zonas grises. Incluso la distinción con su antónimo, las public corporation o publiclyheld corporations, no es del todo clara. Ver NeLSON (2013) p. 1591 nota 105. Las excepciones fundadas en la identidad entre dueños y empresa que caracteriza a esta clase de organizaciones, tienen reconocimiento jurisprudencial y legal. Como ejemplo del primero puede citarse el caso Equal Employment Opportunity Commission v. Townley Engineering \& Manufacturing Co. 859 F.2d 610, 620 (1988) en el que la Corte de Apelaciones del 9o Circuito declaró inoficioso preguntarse acerca de si la empresa tenía libertad religiosa por sí misma, porque al ser un instrumento por el cual los dueños expresan su fe, no tiene derechos diferentes de los que tienen sus dueños. Como ejemplo del segundo puede citarse el estatuto de las $S$ corporations por el que, para efectos tributarios, la ley autoriza a los dueños a declarar las ganancias y pérdidas de la empresa como si fueran propias. Ver http://www.irs.gov/Businesses/Small-Businesses-\&-Self-Employed/S-Corporations [consultada el 27 de diciembre de 2014].

36 El ejemplo paradigmático sería el de las sociedades anónimas abiertas.

37 Es el caso en algunos supuestos del derecho de familia y tributario. Ver Willis (2013) p. 24.

38 Thompson (1991). Un estudio empírico: OH (2010).
} 


\subsection{LA ACTIVIDAD COMERCIAL ¿ES INCOMPATIBLE CON LA RELIGIÓN?}

Un segundo foco de la discusión gira en torno a las implicancias del carácter comercial de las empresas respecto de la legitimidad para invocar el derecho a la libertad religiosa. Es posible distinguir aquí dos niveles distintos, pero complementarios, de la discusión. El primero se refiere a la compatibilidad entre la actividad comercial y la religiosa. El segundo, que viene a ser como una especificación del primero, a la naturaleza de las asociaciones con fines de lucro.

\subsubsection{Las esferas de lo religioso y lo comercial, ¿son compatibles?}

En la base de la posición que rechaza la libertad religiosa de las empresas comerciales, domina la idea de que existe una total incompatibilidad entre religión y negocios, como si la advertencia evangélica "Nadie puede servir a dos señores" (Mt. 6, 24) supusiera una división entre la esfera comercial y la religiosa ${ }^{39}$. Los abogados de esta posición señalan la sentencia de la Corte Suprema United States $v$. Lee como precedente ${ }^{40}$. En particular, invocan el siguiente texto: "Cuando los seguidores de una determinada secta [religiosa] deciden participar en la actividad comercial voluntariamente, los límites que ellos aceptan sobre su propia conducta como consecuencia de sus creencias y opciones religiosas, no pueden superponerse a las regulaciones legales que son obligatorias para las demás personas que participan en la actividad comercial" 41 .

Frente a lo anterior, se ha dicho que la supuesta separación entre la esfera de lo comercial y de lo religioso -y, en definitiva, entre lo secular y lo religioso- es menos nítida de lo que parece. La segunda vertiente de la respuesta destaca la naturaleza permeable de la frontera que media entre la esfera del comercio y la esfera de la religión. Lo hace señalando, en primer lugar, los múltiples ejemplos de empresas comerciales administradas en conformidad con principios de inspiración religiosa ${ }^{42}$. También se ha destacado la doctrina social de la Iglesia Católica, que enseña que la actividad comercial es un camino para vivir la religión ${ }^{43}$. Por último, se invocan algunos casos en que la superposición entre lo secular y lo religioso imposibilita prácticamente que se los trate en forma separada ${ }^{44}$.

\footnotetext{
39 En el fondo de esta objeción, y en la medida que es una proyección de la separación entre los secular y lo religioso, podría verse una determinada comprensión de lo público de tinte rawlsiano. Para esta concepción, el mercado, como lugar de encuentro público, debe estar regulado por razones que sean compartidas por todos los que participan en él. Dado que la religión es algo esencialmente privado, i.e. intransferible racionalmente, está excluida del mercado. Esto se traduce en que la religión no puede ser un argumento válido para imponer o exigir determinadas reglas o conductas en el mundo de los negocios. Un análisis crítico en FINNIS (2011) p. 105 y ss.

40 United States v. Lee, 455 U.S. 252 (1982). El Gobierno Federal propuso este caso en apoyo de su postura, por ejemplo, en Korte v. Sebelius, 735 F.3d 654, 681 (7th Cir. 2013).

41455 U.S. 252, 261 (1982).

42 RienZi (2013) pp. 74 y ss.

43 RiENZI (2013) pp. 69-72. COMPENDIO DE DOCTRINA SOCIAL DE LA IGLESIA, nn. 343 y ss. Disponible en http://www.vatican.va/roman_curia/pontifical_councils/justpeace/documents/rc_pc_justpeace_ doc_20060526_compendio-dott-soc_sp.html\#El papel del empresario y del dirigente de empresa [consultada 25 de enero de 2015].

44 Los casos más recurrentes son Corp. of Presiding Bishop of Church of Jesus Christ of Latter-day Saints v. Amos y Hosanna-Tabor Evangelical Lutheran Church \& Sch. v. E.E.O.C. que se analizan en nota al pie 63 y ss.
} 


\subsubsection{Las empresas con fines de lucro, ¿pueden practicar la religión?}

Quienes objetan la posibilidad de las empresas con fines de lucro para practicar la religión, estarían dispuestos a conceder que una corporación religiosa pueda invocar el derecho a la libertad religiosa pues, al fin y al cabo, sus fines son religiosos. Sin embargo, distinto es el caso de las sociedades comerciales ${ }^{45}$. Así se expresó la mayoría en la sentencia Conestoga: "No vemos cómo una organización con fines de lucro, artificial, invisible, intangible y que existe solo gracias a la ley, y que fue creada para producir dinero, podría ejercer un derecho tan inherentemente humano como la libertad religiosa" ${ }^{36}$. Este tipo de organizaciones persigue fines comerciales y se desenvuelve en el mercado, que es un espacio esencialmente secular o, si se prefiere, neutral, religiosamente hablando ${ }^{47}$.

En contra de esta postura, se afirma una concepción amplia de los fines de las empresas comerciales. Definirlas como "máquinas para producir dinero" 48 es un reduccionismo que apenas refleja un aspecto, y ni siquiera el más significativo, de la realidad abrazada por esta clase de organización. En efecto, son numerosos los ejemplos en que es posible identificar fines que van mucho más allá de ganar de dinero ${ }^{49}$. Más aún, es prácticamente imposible encontrar alguna empresa cuya misión sea, al menos explícitamente, ganar dinero.

\subsection{Sobre la RELEVANCIA DE CIERTAS Distinciones}

El tercer argumento sostiene que, si se admite que RFRA es aplicable también a las corporaciones con fines de lucro, se perderá la distinción entre lucro/no-lucro y entre secular/religioso. Y es importante conservar estas distinciones, según sus defensores. Un primer ámbito en donde esto podría ocurrir es en el de la aplicación del HHS Mandate ${ }^{50}$. Otro ámbito en el que reconocer libertad religiosa a las empresas comerciales podría tener consecuencias negativas es el de los impuestos federales. La sección $\$ 501$ del Internal Revenue Code establece exenciones tributarias para organizaciones religiosas y para organizaciones sin fines de lucro ${ }^{51}$. El argumento postula que las corporaciones con fines de lucro, puesto que no califican para las exenciones de la sección $\$ 501$, carecen de legitimidad para invocar RFRA ${ }^{52}$. Esta distinción $-y$ sus efectos- se vería debilitada si la aplicación de RFRA

\footnotetext{
45 Es la tesis de NeLSON (2013).

46 Conestoga Wood Specialties Corp. v. Secy of U.S. Dep't of Health \& Human Servs., 724 F.3d 377, 385 (3d Cir. 2013).

47 Colombo (2013) pp. 6 y 7, notas al pie números 21 a 27.

48 Greenfield et al. (2007) p. 883.

49 Colombo (2013) pp. 16 y ss.

50 Así lo entendió una corte de distrito en la sentencia O’Brien v. U.S. Dep’t of Health \& Human Servs., 894 F. Supp. 2d 1149, 1164 (E.D. Mo. 2012): “exceptuar a las corporaciones religiosas [del HHS Mandate] es posible porque trazar una línea (...) entre lo religioso y lo secular es constitucionalmente permisible. La excepción en favor de un empleador religioso implica necesariamente la distinción entre empleadores religiosos y seculares, y el HHS Mandate ha trazado un línea lógica y clara entre los dos”. Se sigue que si la excepción se ampliara a los empleadores seculares, el HHS Mandate dejaría de ser la regla.

51 Cfr. 26 U.S.C.A. $\$ 501(\mathrm{a})(\mathrm{c})(3)(4)(\mathrm{A})(\mathrm{B})$

52 La Corte de Apelaciones del 10 Circuito ironiza en Hobby Lobby Stores, Inc. v. Sebelius, 723 F.3d 1114 , 1134 (10th Cir. 2013): “aparentemente, el problema para el Gobierno consiste en las corporaciones que no satisfacen la sección $\$ 501$ (c)(3) del Internal Revenue Code. Cuando eso ocurre, de alguna manera el derecho a la libertad religiosa desaparece".
} 
se extendiera a corporaciones que, por su carácter comercial, se suponen excluidas del alcance original de la ley. Un tercer ámbito sería el de las excepciones por motivos religiosos limitadas a cierta clase de corporaciones. Importantes disposiciones legales descansan en la distinción profitlnon-profit y secular/religioso. Tres ejemplos significativos son la Civil Rights Act ${ }^{53}$, la Americans with Disabilities Act (ADA) ${ }^{54}$ y la National Labor Relations Act (NLRA) ${ }^{55}$. A la luz de estas disposiciones, la aplicación de RFRA para eximir a las corporaciones con fines de lucro de un deber legal, podría acarrear serias consecuencias en la aplicación de las leyes antidiscriminación. El peligro anunciado por el Gobierno puede formularse así: si una empresa comercial puede ser eximida de pagar ciertas prestaciones de salud a sus empleadas por motivos religiosos, ¿por qué no podría ser eximida de contratar o despedir empleadas por motivos religiosos?

A las anteriores objeciones se ha contestado señalando que las distinciones entre lucro/no-lucro y secular/religioso son referenciales, no dispositivas. Aunque sea una perogrullada, conviene hacer notar que este par de binomios son semánticamente diferentes. Asumir una identificación entre lucro y secular, por una parte, y entre no-lucro y religioso, por otra, sería un error. En realidad, los dos pares de categorías pueden combinarse entre sí dando lugar a cuatro resultados diferentes ${ }^{56}$. Respecto de la dicotomía lucro/no-lucro que se supone amenazada por el eventual reconocimiento de la libertad religiosa de las corporaciones comerciales, Willis ofrece varias razones para concluir que "es simplista y equívoca" 57 . En primer lugar, la distinción no está precisamente establecida con carácter general en ley alguna. Cuando algunas leyes de los Estados echan mano de ella, la distinción no se refleja en ninguna diferencia substancial entre las dos clases de corporaciones ${ }^{58}$. En relación al Internal Revenue Code, la distinción no tiene efectos reales en la tributación. Las

\footnotetext{
${ }^{53}$ La discriminación laboral por motivos religiosos está, en general, prohibida; pero entre las excepciones se considera a las corporaciones religiosas. Dice la ley: "[This anti-discrimination law] shall not apply to (...) a religious corporation, association, educational institution, or society with respect to the employment of individuals of a particular religion to perform work connected with the carrying on by such corporation, association, educational institution, or society of its activities". 42 U.S.C.A. $\$ 2000 \mathrm{e}-1$ (West).

54 Cfr. 42 U.S.C.A. $\$ 12113$ (West).

55 La interpretación del Gobierno fue que, conforme al precedente establecido por NLRB v. Catholic Bishop, 440 U.S. 490 (1979), la ley asignaría una importancia crucial a la distinción entre corporaciones religiosas y no religiosas. Pero en dicho caso la distinción solo fue útil para fijar la competencia de la National Labor Relations Board pues, como señaló la Corte Suprema, esta agencia no tiene jurisdicción sobre colegios operados por la Iglesia.

${ }^{56}$ En el caso de las corporaciones, es posible que estas sean religiosas con fines de lucro, seculares sin fines de lucro, religiosas sin fines de lucro y seculares con fines de lucro. A esto hizo alusión la Corte de Apelaciones del $10^{\circ}$ Circuito cuando dijo que, "aunque el Gobierno cree que la distinción lucro/no-lucro deriva de la natural diferencia entre negocios y religión, nosotros observamos que la variedad de formas que una corporación puede adoptar no refleja esa supuesta natural diferencia”. Hobby Lobby Stores, Inc. v. Sebelius, 723 F.3d 1114, 1135 (10th Cir. 2013).

57 Willis (2013) p. 61.

58 Willis (2013) p. 61. Por ejemplo, la diferencia puede ser relevante para el caso de una fusión; la ley del Estado de Nueva Jersey prohíbe la fusión entre una corporación sin fines de lucro y una con fines de lucro (N.J. Stat. Ann. \$15A:10-1) También puede ser relevante en el caso de disolución. Ver GERSTENBLITH (2006) p. 228. TAN (2013) p. 1357 asume que unas y otras corporaciones no son idénticas, pero la pregunta es "en que se diferencia respecto de la libertad religiosa". Concluye que en este aspecto no difieren, porque "muchas organizaciones de beneficencia (non-profit) lucran".
} 
exenciones tributarias favorecen a las organizaciones de beneficencia, pero ninguna norma exige que dichas asociaciones renuncien al lucro o se organicen $\mathrm{u}$ operen conforme a las leyes que regulan a las corporaciones sin fines de lucro ${ }^{59}$. En segundo lugar, numerosas organizaciones sin fines de lucro tienen ingresos económicos como resultado de su actividad. Colegios y hospitales son los casos más comunes ${ }^{60}$. A esto debe sumársele el margen que tienen para realizar operaciones esencialmente comerciales ${ }^{61}$. En tercer lugar, la amplia variedad de formas que puede adoptar una corporación sin fines de lucro torna prácticamente inútil la distinción, si se quiere hacer uso de ella en abstracto y apriorísticamente ${ }^{62}$. Respecto de la distinción secular/religioso contenida en las distintas leyes antidiscriminación, Rienzi explica que en ninguna de ellas la distinción supone una incompatibilidad entre el carácter religioso de una corporación y la naturaleza supuestamente secular de la actividad comercial $^{63}$.

Un ejemplo que ilustra lo frágil de la distinción secular/religioso es Corp. of Presiding Bishop of Church of Jesus Christ of Latter-day Saints v. Amos ${ }^{64}$. En este caso, un empleado que trabajaba en un gimnasio de propiedad de la Iglesia de Jesucristo de los Santos de los Últimos Días fue despedido porque, al no estar certificado como miembro de dicha Iglesia, carecía de un requisito necesario para cuidar de sus templos. El afectado demandó a su empleador denunciando discriminación religiosa: puesto que su trabajo como ingeniero eléctrico era de naturaleza secular, alegó, no debía estar condicionado a cualificaciones religiosas. En primera instancia la corte de distrito acogió la demanda. Dijo que eximir de responsabilidad al empleador en un caso como este llevaría a que "iglesias con recursos financieros extendieran, de manera impermisible, su influencia y propagaran su fe en el mundo de los negocios a través de la actividad comercial" ${ }^{65}$. Sin embargo, la Corte Suprema revirtió el fallo, fundamentalmente porque la distinción entre actividades seculares y religiosas es poco clara ${ }^{66}$. Justice O’Connor en su voto concurrente fue más lejos, y declaró

\footnotetext{
59 WiLlis (2013) p. 63.

60 Willis (2013) p. 63. Rienzi (2013) p. 39 aclara que la distinción lucro/no-lucro, en el contexto de las exenciones tributarias, "no sugiere ningún tipo de regla que prescriba categóricamente que la religión y el lucro no puedan coexistir".

${ }^{61}$ El margen no puede exceder el 15\% del total de todas sus actividades. Willis (2013) p. 64.

${ }^{62}$ Willis (2013) p. 66. Por su parte, BERG (2006) p. 165, considerando la utilidad de la distinción nonprofit/ for-profit respecto de la protección de la libertad religiosa, concluye que es problemática en muchos casos relevantes y que solo sirve para responder un limitado rango de cuestiones". RIENZI (2013) p. 56 muestra como "para efectos de la aplicación de la Constitución, las cortes han rechazado rutinariamente los argumentos basados en distinciones categóricas entre corporaciones con y sin fines de lucro”.

63 RiEnZI (2013) p. 58.

64483 U.S. 327, 107 S. Ct. 2862, 97 L. Ed. 2d 273 (1987)

65 Amos, 483 U.S. 327, 337, 107 S. Ct. 2862, 2869, 97 L. Ed. 2 d 273 (1987).

66 Justice White expresó que "la línea que las separa está lejos de ser clara”. Amos, 483 U.S. 327, 336. Agregó que la excepción a la Civil Rights Act contemplada en la sección 702 del Título VII, fue diseñada por el Congreso para cubrir todas las actividades del empleador religioso". Amos, 483 U.S. 327, 339 (énfasis agregado). Justice Brennan dijo al respecto: "Lo que hace difícil aplicar la distinción religioso/secular es que el carácter de una actividad determinada no es evidente”. Amos, 483 U.S. 327, 343.
} 
abierta la cuestión acerca de si la excepción del Título VII de la Civil Rights Act protege las actividades comerciales de las corporaciones religiosas ${ }^{67}$.

\subsection{Libertad RELigiosa de las PERSONAS JURÍdicAs: ¿PROTEGER A UNAS (COMERCIALES) Y DESPROTEGER A OTRAS (RELIGIOSAS)?}

Otro riesgo implícito en el reconocimiento de la libertad religiosa de las personas jurídicas con fines de lucro es el debilitamiento de la protección de las demás asociaciones. Si la naturaleza de la asociación fuera totalmente indiferente para efectos de proteger la libertad religiosa, las corporaciones religiosas podrían verse perjudicadas por la aplicación de un estándar más estricto, adecuado a la realidad de las empresas comerciales. Robert K. Vischer llama la atención sobre este punto, advirtiendo el peligro que supone obviar totalmente la naturaleza del ente que reclama protección ${ }^{68}$. Desde la perspectiva de la libertad religiosa, sería un error tratar a las empresas y a las iglesias como si fueran iguales, aunque las dos estén organizadas como personas jurídicas ${ }^{69}$. Vischer no afirma explícitamente que las empresas comerciales están excluidas de RFRA, pero en cambio previene de los peligros de una interpretación demasiado amplia de la ley, que pase totalmente por alto la naturaleza y características de la corporación que reclama. Gaylord sostiene precisamente esto último. Para él, First Nat. Bank of Boston v. Bellotti ${ }^{70}$ y Citizens United v. Fed. Election Comm'n $n^{71}$ sientan los precedentes para una aplicación de RFRA completamente indepen-

${ }^{67}$ Amos, 483 U.S. 327, 349. Justice Blackmun concurrió en este punto con O'Connor. Un caso reciente que ilustra la misma dificultad es Hosanna-Tabor Evangelical Lutheran Church \& Sch. v. E.E.O.C., en el que una profesora de un colegio protestante fue despedida porque, de acuerdo a sus empleadores, no observó la conducta esperable de quienes sirven en esa congregación. La profesora demandó alegando discriminación religiosa, porque su trabajo como profesora era enseñar materias esencialmente seculares. Para decidir este caso, la calificación de la condición de la profesora era determinante. Pero la Corte Suprema rehusó entrar en esa materia, dado lo difuso de la línea que separa lo secular de lo religioso. La Corte explicó que la distinción entre actividades seculares y religiosas de una profesora que trabaja para una corporación religiosa es irrelevante para efectos de la excepción del Título VII, si es que es posible hacerla; el hecho es que "los mismos líderes de las congregaciones religiosas tienen una combinación de deberes, entre ellos muchos de naturaleza secular, tales como ayudar en la administración financiera de la congregación, en la supervisión del personal estrictamente secular, y en el seguimiento de la mantención de los edificios". En el mismo sentido, DurHam y Sewell (2006) p. 60.

${ }^{68}$ VisCher (2013) pp. 389 y 398.

${ }^{69}$ Vischer apunta que, "en términos de la posición que ocupan respecto de la identidad religiosa de la mayoría de los Americanos, [las empresas comerciales] palidecen en comparación con la posición central de iglesias y organizaciones benéficas". VISCHER (2013) p. 396.

70435 U.S. 765 (1978). En este caso la Corte Suprema sostuvo la inconstitucionalidad de una ley estatal que prohibía a las empresas contribuir con dinero para hacer campaña en referéndums, porque las empresas también gozan de la libertad de expresión protegida por la Primera Enmienda de la Constitución.

71558 U.S. 310 (2010). Un fuerte argumento en favor de la libertad religiosa de las corporaciones con fines de lucro se basa en la decisión Citizens United. En este caso, la Corte suprema declaró la inconstitucionalidad de una ley federal que prohibía a las corporaciones usar sus fondos para financiar discurso político (political speech). La sentencia sostuvo que "el Gobierno no puede suprimir la libertad para expresarse políticamente (political speech) basándose en el carácter corporativo de quien habla. No existe por parte del Gobierno un interés que pudiera justificar la limitación de esta libertad a las corporaciones con o sin fines de lucro". Este precedente, en el contexto del HHS Mandate y RFRA, significa que la protección de la libertad religiosa no debiera hacer ninguna distinción basándose en la naturaleza de la asociación. Si es que la protección de la libertad para expresarse políticamente (political speech) es protegida con independencia de la naturaleza del demandante, ¿por 
diente de la naturaleza y características de quien reclama. Sin embargo, estos dos precedentes, en realidad, están lejos de ser decisivos en el contexto del HHS Mandate ${ }^{72}$. Aunque es cierto que ambas sentencias declararon que la naturaleza de la corporación era irrelevante para adjudicar el derecho fundamental en conflicto, en los dos casos se trataba de la libertad de expresión. Y esta circunstancia los distingue sustancialmente del caso de la libertad religiosa ${ }^{73}$. Por lo tanto, incurriría en un error quien postulara que los fundamentos de $B e-$ llotti y Citizens United son los mismos que deberían sostener una legitimación irrestricta de las empresas comerciales para demandar la protección de la libertad religiosa.

El riesgo de que la ampliación del espectro de RFRA perjudique el estándar de protección de las corporaciones religiosas es insignificante, porque solo las empresas del tipo close corporations se benefician de dicha ampliación ${ }^{74}$. Dado el estrecho grado de identificación entre dominio y administración que caracteriza a las close corporations, la libertad religiosa de estas asociaciones es, en términos prácticos, la misma de sus dueños y administradores. El factor que mantiene intacto el estándar de protección de RFRA e impide su disolución pese a la incorporación de las empresas comerciales, es el test de la sinceridad con que se afirman las convicciones religiosas para las que se reclama protección. Para aplicar RFRA los jueces deben estar convencidos de que el demandante sinceramente cree lo que dice creer $^{75}$. Las dificultades para probar este elemento son bien conocidas y existe un razonable margen de error ${ }^{76}$. Pese a todo, su satisfacción obliga a que, en definitiva, sea la conducta de individuos específicos, expresada a través de la administración de la empresa, lo que se pondere judicialmente ${ }^{77}$. Y esto no difiere de lo que ha sido el cauce normal de

qué habría de hacerse la distinción cuando se trata de la libertad religiosa? COLOMBO (2013) pp. 69 y ss., sostiene esta posición.

${ }^{72}$ GAYLORD (2014) p. 648 sostiene lo contrario. En su opinión, Bellotti y Citizens United obviaron la naturaleza del actor porque lo relevante en la decisión era la actividad para la que se reclamaba protección. Del mismo modo, en el caso del HHS Mandate, lo relevante no es quien reclama, sino si lo que se reclama está protegido por la Constitución o no. WHeLAn (2012) pp. 2182-2183, demostraría que objetar el cumplimiento del HHS Mandate por motivos religiosos estaría protegido por la Primera Enmienda.

${ }^{73}$ La premisa que subyace a la protección de la libertad de expresión (el discurso político, en este caso), es que mientras más sean las fuentes de expresión, mejor para la Sociedad. Se entiende que "el mercado de las ideas" facilita la consecución de bienes sociales valiosos, como la búsqueda de la verdad, el control de los abusos gubernamentales o la promoción de la deliberación democrática. SCHAUER (2005). La protección de la libertad religiosa no debe plantearse desde la premisa de la libertad de expresión, porque la religión no cumple la misma función.

${ }^{74}$ Esta no es una afirmación de principio, sino de hecho. Por una parte, hasta ahora todas las corporaciones con fines de lucro que han reclamado la aplicación de RFRA contra el HHS Mandate han sido close corporations (ver http://www.becketfund.org/hhsinformationcentral/ Consultado el 21 de julio de 2014). Por otra parte, las probabilidades de que publicly-held corporations demanden la aplicación de RFRA son prácticamente inexistentes. En primer lugar, por las obvias dificultades para determinar la voluntad corporativa en tan delicada materia. En segundo lugar, por la brecha que separa el dominio de la empresa de su diaria administración. Tercero, por el relativamente bajo número de estas empresas en comparación con el universo total de las corporaciones con fines de lucro. Ver infra nota 77.

75 Es el llamado sincerity test, inaugurado en la sentencia United States v. Ballard, 322 U.S. 78 (1944) y ratificado en United States v. Seeger, 380 U.S. 163 (1965).

76 Greenawalt (1998).

77 Justice Briscoe admite que la distinción entre corporaciones publicly-held/closely-held es relevante solo en cuanto permite tener en cuenta las creencias religiosas de los dueños y fundadores de las [close] corporations". Hobby Lobby Stores, Inc. v. Sebelius, 723 F.3d 1114, 1173 (10th Cir. 2013). 
aplicación de RFRA. Luego, si el estándar de protección de las corporaciones religiosas no se ha visto perjudicado hasta ahora, no hay razones para pensar que lo será en el futuro, como consecuencia de atribuir a las close corporations el derecho a la libertad religiosa.

\section{5. ¿AUMENTARÁ EL VOLUMEN DE LITIGACIÓN?}

Existen fundamentos empíricos para pensar que habrá un aumento significativo en la litigación una vez reconocido el derecho de las empresas comerciales para invocar la libertad religiosa: el comercio en los Estados Unidos prácticamente depende de las corporaciones con fines de lucro y, más específicamente, de empresas de características similares a aquellas que han demandado contra el HHS Mandate ${ }^{78}$. Aunque las empresas afectadas por el HHS Mandate sean pocas comparadas con el total de empresas, el número de ellas es todavía significativo ${ }^{79}$. Teóricamente, si todas ellas de hecho reclamaran una excepción por motivos religiosos, el resultado sería desastroso desde el punto de vista de su gestión en tribunales. Por otra parte, aceptar argumentos religiosos para eximir a las corporaciones con fines de lucro del cumplimiento de una obligación legal, puede abrir las puertas para que, por la misma clase de motivos, pidan eximirse de otras obligaciones legales en el futuro ${ }^{80}$. En su voto disidente, Justice Briscoe teme que con el mismo argumento con que se exime hoy a las empresas de pagar por anticonceptivos, en el futuro se exima "a un empresario Testigo de Jehová de pagar las transfusiones de sangre del plan de salud de sus empleados, o a corporaciones de dueños católicos para que priven a sus empleados de cobertura para hospicios o histerectomías, o para que empresarios miembros de la Cienciología puedan rehusar la cobertura de antidepresivos o tratamientos psiquiátricos de emergencia. Y para que ciertos musulmanes, judíos e hindúes puedan, a través de sus corporaciones, rehusar la cobertura de medicamentos u otros que contengan productos derivados del cerdo o la vaca" ${ }^{\text {. }}$

El reconocimiento de la libertad religiosa de las corporaciones con fines de lucro no aumentará la litigación. En primer lugar, habría que decir que el eventual incremento de los índices de litigación como consecuencia del establecimiento de un nuevo criterio de decisión, no es un argumento en contra de la aplicación de dicho criterio. Aunque consideraciones como estas no son, ni deben ser, absolutamente ajenas al razonamiento para decidir un caso, jamás deberían prevalecer sobre las consideraciones estrictamente jurídicas (o de derecho, o de justicia, como se prefiera). No obstante lo anterior, la preocupación por un aumento en la litigación solo tendría fundamentos si es que se asume que la libertad reli-

\footnotetext{
78 El 95\% de los negocios en los Estados Unidos corresponde a empresas en que la propiedad y la administración se superponen significativamente (close corporations). WEBER (2012) p. 218.

79 Aparte de las corporaciones religiosas que se benefician de la excepción, están excluidas de la aplicación del HHS Mandate las empresas con menos de 50 empleados full-time y las empresas con planes contratados hasta el 23 de marzo de 2010 (grandfathered plans), siempre y cuando estos no se modifiquen.

80 Es la opinión de Justice Briscoe en su voto disidente: "Si es que todo lo que una corporacion requiere para ser categorizada como faith-based business, para efectos de RFRA, es una combinación de amplias declaraciones de contenido religioso en la misión corporativa con unas creencias religiosas más específicas por parte de los dueños o fundadores de la corporación, (...) entonces se estarían abriendo las compuertas para que a través de RFRA se cuestionaran una serie de leyes federales que gobiernan los asuntos corporativos". Hobby Lobby Stores, Inc. v. Sebelius, 723 F.3d 1114, 1174 (10th Cir. 2013).

81 Hobby Lobby Stores, Inc. v. Sebelius, 723 F.3d 1114, 1174 (10th Cir. 2013) nota al pie n.8.
} 
giosa de los individuos es anulada o severamente limitada desde el momento en que se asocian por propósitos comerciales. En caso contrario, negar a las corporaciones legitimación para reclamar la aplicación de RFRA no prevendrá una escalada en la litigación, porque los individuos detrás de esas corporaciones siempre estarán legitimados para requerir la aplicación de RFRA. Por último, el temor al aumento de la litigación como consecuencia de extender los derechos fundamentales a las corporaciones sería históricamente infundado ${ }^{82}$.

\section{LA SENTENCIA HOBBY LOBBY}

La Corte Suprema decidió que las empresas comerciales, al menos aquellas que pueden ser calificadas como close corporations, eran titulares del derecho a la libertad religiosa y, por lo tanto, merecedoras de la protección que RFRA asegura a toda persona ${ }^{83}$. Esta decisión descansa en dos premisas: (4.1) la forma societaria no inhibe el derecho de los asociados a la libertad religiosa, y (4.2) la actividad comercial no es incompatible con la religión.

4.1. La sentencia reconoce que la persona jurídica es un sujeto de derecho distinto de las personas naturales vinculadas a ella, pero corrige el argumento del Gobierno, i.e. al asociarse, las personas naturales no pueden proyectar su libertad religiosa a través de la asociación. La Corte Suprema hace ver que la distinción no debe entenderse como separación. En definitiva, dice la Corte, "cuando la ley o la Constitución protegen los derechos de las personas jurídicas, lo hacen para proteger los derechos de las personas naturales vinculadas a ellas" ${ }^{84}$. En la fundamentación de este punto, la Corte lleva hasta el extremo el argumento aquel de que las personas jurídicas no pueden "realizar ninguna acción de inspiración religiosa de manera independiente y separada de la intención y dirección de los individuos que actúan en su nombre". En realidad, dice la Corte, las personas jurídicas separadas de las personas naturales, no pueden hacer absolutamente nada ${ }^{85}$. Por lo mismo, invocar la distinción entre la empresa y sus dueños para explicar la limitación de los derechos de estos últimos desconoce el fundamento básico de todos los derechos de que gozan las empresas (o cualquier otra organización). En último término, "una corporación es sencillamente una forma de organización que los seres humanos utilizan para alcanzar determinados fines" ${ }^{\text {" }}$. Sería absurdo, por lo tanto, que al asociarse los seres humanos vieran limitados sus derechos, y quizá precisamente aquellos derechos para cuya más plena satisfacción se asociaron. La Corte no ignora que el argumento puede perder fuerza si se trata de aplicarlo a empresas

82 Cuando la Corte Suprema decidió, en Santa Clara Cnty. v. S. Pac. R. Co., 118 U.S. 394, 6 S. Ct. 1132,30 L. Ed. 118 (1886) que las corporaciones eran personas para efectos de las 14a Enmienda de la Constitución, sentando así el fundamento de las sucesivas atribuciones de otros derechos fundamentales, había mejores motivos para temer un aumento en la litigación. Sin embargo, este no se produjo. Cfr. Colombo (2013) p. 52 o WeBER (2012) p. 222.

83 Por este motivo, la Corte Suprema consideró innecesario resolver si acaso la libertad religiosa de las empresas comerciales está protegida también por la Primera Enmienda de la Constitución. Cfr. Burwell v. Hobby Lobby, p. 49.

${ }^{84}$ Burwell v. Hobby Lobby, p. 18

85 Burwell v. Hobby Lobby, p. 19

86 Burwell v. Hobby Lobby, p. 18 
con cientos o miles de accionistas, como IBM o General Electric. En casos como estos, la fuerza y extensión del vínculo entre las personas y la empresa se debilita y restringe. Además, se complica la posibilidad de identificar la voluntad de la empresa con la voluntad de sus dueños. Sin embargo, puesto que esta no es la situación de Hobby Lobby Stores Inc., la Corte no se siente llamada a resolver el problema que esa clase de empresa podría ofrecer ${ }^{87}$.

4.2. La sentencia aborda la relación entre las esferas comercial y religiosa en tres niveles, por así decirlo. En el primero, establece que existe una cierta superposición entre ambas, al punto que se afectan mutuamente. En el segundo, defiende la compatibilidad entre la actividad comercial y la práctica de la religión. En el tercero, afirma la complementariedad entre ambas, al menos en el caso de las empresas. En cuanto a la intersección entre los ámbitos del comercio y la religión, la Corte trae a colación la sentencia Braunfeld $v$. Brown, en la que se rechazó la demanda de un grupo de comerciantes judíos contra el Estado de Pennsylvania por establecer que el comercio debía permanecer cerrado durante el domingo ${ }^{88}$. Allí quedó establecido que una ley que implica -aun indirectamente- costos económicos para una persona en razón de su fe, es una ley que afecta su libertad religiosa ${ }^{89}$. Acerca de la compatibilidad entre el mundo de los negocios y la fe, la Corte vuelve a recurrir a la sentencia Braunfeld. Cita este caso de 1961 para demostrar que la dedicación a los negocios nunca ha sido impedimento para reclamar ante los tribunales el reconocimiento de la libertad religiosa. Otra cosa es ganar el caso, por supuesto; pero de lo que aquí se trata es de probar que la actividad comercial no priva de legitimidad activa a quienes participan en ella. Por último, en cuanto a la complementariedad de las dos esferas, la Corte reconoce la legitimidad de organizarse como una empresa comercial para perseguir fines de naturaleza religiosa. Esta complementariedad descansa en una concepción amplia de las sociedades comerciales. La Corte recuerda que lo único que exige el Derecho para otorgar a una organización el estatus de persona jurídica con fines de lucro es que persiga una finalidad que no sea ilegal $^{90}$. Y de hecho, constata la Corte, hay una pluralidad de fines que pueden orientar la actividad de la empresa. Luego, concluye, "si una corporación con fines de lucro puede perseguir fines valiosos [distintos del lucro, tales como la promoción de objetivos humanitarios o altruistas], no hay ninguna razón evidente para que no puedan perseguir fines religiosos también" $"$. Con esto la Corte echa por tierra la concepción de las organizaciones con fines de lucro como sinónimo de entes maximizadores de utilidades. Pueden existir una serie de ventajas asociadas al estatus de empresa comercial, distintas del lucro, que justifiquen la decisión de organizarse así.

La sentencia Hobby Lobby, que fue la última del año judicial 2013-2014², provocó apasionadas reacciones en las dos orillas del conflicto. Este caso simbolizaba un episodio

\footnotetext{
${ }^{87}$ Burwell v. Hobby Lobby, p. 29

88 Braunfeld v. Brown, 366 U.S. 599 (1961)

89 Burwell v. Hobby Lobby, p. 21.

90 Burwell v. Hobby Lobby, p. 23

91 Burwell v. Hobby Lobby, p. 23

92 No es raro que la Corte Suprema reserve para el último día del año judicial la decisión de los casos más polémicos.
} 
más de la "guerra cultural" que enfrenta a liberales y conservadores en los Estados Unidos Por supuesto que la decisión se ha considerado como una victoria conservadora, en parte porque representa un espaldarazo a la religión, y, en parte, porque se la entiende como un gesto hacia el movimiento pro-life ${ }^{94}$. El Gobierno federal ha propuesto una nueva redacción del HHS Mandate acorde con los criterios definidos en la sentencia ${ }^{95}$, y las cortes inferiores están obligadas a utilizar el precedente por ella establecido.

\section{CONCLUSIÓN}

La sentencia Burwell v. Hobby Lobby establece un precedente cuyo interés trasciende las fronteras de los Estados Unidos. Esto por un doble motivo: por el contenido mismo del nuevo precedente y por los supuestos en que descansa. Al afirmar la legitimidad de las empresas comerciales -al menos de aquellas en que la propiedad está concentrada en pocas personas- para invocar el derecho a la libertad religiosa contra el Gobierno federal, la Corte Suprema precisó el contenido de la libertad religiosa tal y como está protegida por RFRA. De paso, reconoció el carácter instrumental de las asociaciones, comerciales o no, en función de la mayor realización de los seres humanos, y declaró la compatibilidad entre religión y negocios.

El interés de la sentencia alcanza a Chile, porque el conflicto por ella resuelto ya se ha presentado en nuestro país y, seguramente, se volverá a presentar. Es probable que si, en 2007, las farmacias hubiesen conocido los argumentos discutidos en este caso, habrían echado mano de ellos para reclamar una excepción a la obligación general de vender la píldora: los dueños habrían podido legítimamente reclamar que su religión no era una cuestión ajena a las políticas de su empresa. De igual modo, es plausible que en el futuro, los argumentos debatidos con ocasión de la ejecución del HHS Mandate, sean útiles a quienes vean la práctica de su religión amenazada por el Gobierno a través de leyes dirigidas a las personas jurídicas.

\section{BIBLIOGRAFÍA}

Benedict, Kathryn S. (2013): "When Might Does Not Create Religious Rights: For-Profit Corporations' Employees and the Contraceptive Coverage Mandate", Columbia Journal of Gender and Law, vol. 26, Issue 1: pp. 58-122.

\footnotetext{
93 Lupu (2014) p. 4 y ss.

94 Susan Berry, Pro-Life Community Celebrates Scotus Hobby Lobby Decision, 30 de junio de 2014, en http:// www.breitbart.com/big-government/2014/06/30/pro-life-community-celebrates-scotus-decision-for-religiousfreedom-against-obamacare-hhs-abortion-pill-mandate/ [consultada el 27 de diciembre de 2014]. Así también se entendió por alguno en Chile, lo cual replicó a escala local un aspecto de la discusión generada en los Estados Unidos. Me refiero al debate habido entre Hernán Corral y varios profesores a través de las Cartas al Director de El Mercurio, entre el 16 de julio y el 15 de agosto de 2014.

95 Federal Register, vol. $79 \mathrm{~N}^{\circ} 166,27$ de agosto de 2014, 51118 y ss.
} 
Berg, Thomas C. (2006): "Religious Structures under the Federal Constitution", en James A. Serritella et al. (edits.), Religious Organizations in the United States: A Study of Identity, Liberty, and the Law (Carolina Academic Press) pp. 129-166.

Colombo, Ronald J. (2013): “The Naked Private Square”, Houston Law Review, vol. 51, Issue 1, pp. 1-88.

Durham, Cole, Jr. and Sewell, Elizabeth A. (2006): "Definition of Religion”, en James A. Serritella et al. (edits.), Religious Organizations in the United States: A Study of Identity, Liberty, and the Law (Carolina Academic Press) pp. 3-84.

Finnis, John (2011): Collected Essays, Vol. V, (Oxford, Oxford University Press) 450 pp.

Gaylord, Scott W. (2014): "For-Profit Corporations, Free Exercise, and the HHS Mandate”, Washington University Law Review, vol. 91, Issue 3: pp. 589-658.

Gerstenblith, Patty (2006): "Associational Structures of religious Organizations", en James A. Serritella et al. (edits.), Religious Organizations in the United States: A Study of Identity, Liberty, and the Law (Carolina Academic Press) pp. 223-252.

Greenawalt, Kent (1998): "Judicial Resolution of Issues about Religious Conviction”, Marquette Law Review, vol. 81, Issue 2: pp. 461-472.

Greenfield, Kent; Greenwood, Daniel J.H.; Jaffe, Erik S. (2007): "Should Corporations Have First Amendment Rights”, Seattle University Law Review, vol. 30, Issue 4: pp. 875-894.

Hall, Matthew I. y Means, Benjamin (2014): "The Prudential Third-Party Standing of Family-Owned Corporations", University of Pennsylvania Law Review Online, vol. 162: pp. 151-164.

Kartchner, Andrew B. (2014): "Corporate Free Exercise: A Survey of Supreme Court Cases Applied to a Novel Question”, Regent Journal of Law \& Public Policy, vol. 6, Issue 2: pp. 85-118.

Lupu, Ira C. (2014): "Hobby Lobby and the Dubious Enterprise of Religious Exemptions", Harvard Journal of Law and Gender, vol. 38, No. 1, (Symposium, Religious Accommodation in the Age of Civil Rights), disponible en SSRN: http://ssrn.com/ abstract $=2466571$

Mayer, Carl J. (1990): "Personalizing the Impersonal: Corporations and the Bill of Rights”, Hastings Law Journal, vol. 41, Issue 3: pp. 577-668.

Miller, Darrell A. H. (2011): "Guns, Inc.: Citizens United, McDonald, and the Future of Corporate Constitutional Rights”, New York University Law Review, vol. 86, Issue 4: pp. 887-957.

Nelson, James D. (2013): “Conscience, Incorporated", Michigan State Law Review, vol. 2013, Issue 5: pp. 1565-1620.

OH, Peter B. (2010): "Veil-Piercing”, Texas Law Review, vol. 89, Issue 1: pp. 81-146.

RienzI, Mark L. (2013): "God and the Profits: Is There Religious Liberty for Moneymakers", George Mason Law Review, vol. 21, Issue 1: pp. 59-116.

Rutledge, Thomas E. (2014): "A Corporation Has No Soul - The Business Entity Law Response to Challenges to the PPACA Contraceptive Mandate", William and Mary Business Law Review, vol. 5, Issue 1: pp. 1-54. 
SCHAUER, Frederick (2005): “Towards an Institutional First Amendment”, Minnesota Law Review, vol. 89, Issue 5: pp. 1256-1279.

TaN, Jonathan T. (2013): "Nonprofit Organizations, for-Profit Corporations, and the HHS Mandate: Why the Mandate Does Not Satisfy RFRA's Requirements", University of Richmond Law Review, vol. 47, Issue 4: pp. 1301-1372.

Thompson, Robert B. (1991): "Piercing the Corporate Veil: An Empirical Study", Cornell Law Review, vol. 76, Issue 5, pp. 1036-1074.

VisCher, Robert K (2013): “Do For-Profit Business Have Free Exercise Rights”, Journal of Contemporary Legal Issues, vol. 21: pp. 369-400.

Weber, Kyle J. (2012): "Corporate Personhood and the First Amendment: A Business Perspective on an Eroding Free Exercise Clause", Rutgers Journal of Law and Religion, vol. 14, Issue 1, pp. 217-248.

Whelan, Edward (2012): “The HHS Contraception Mandate vs. the Religious Freedom Restoration Act”, Notre Dame Law Review, vol. 87, Issue 5, pp. 2179-2190.

Willis, Steven J. (2013): "Corporations, Taxes, and Religion: The Hobby Lobby and Conestoga Contraceptive Cases", South Carolina Law Review, vol. 65, Issue 1, pp. 1-80.

Woytash, John (1978): "We Must Stop Viewing Corporations as People", American Bar Association Journal, vol. 64: p. 814.

\section{JURISPRUDENCIA CITADA}

(todos los fallos corresponden al sistema judicial federal de los Estados Unidos)

Cortes DE DisTRITO

Hobby Lobby Stores, Inc. v. Sebelius, 870 F. Supp. 2d 1278, 1291 (W.D. Okla. 2012)

O'Brien v. U.S. Dep't of Health \& Human Servs., 894 F. Supp. 2d 1149, 1164 (E.D. Mo. 2012)

Cortes de Apelaciones

Equal Employment Opportunity Commission v. Townley Engineering \& Manufacturing Co. 859 F.2d 610 (9th Cir. 1988)

Hobby Lobby Stores, Inc. v. Sebelius, 723 F.3d 1114 (10th Cir. 2013)

Korte v. Sebelius, 735 F.3d 654, 681 (7th Cir. 2013).

Conestoga Wood Specialties Corp. v. Secy of U.S. Dep't of Health \& Human Servs, 724 F.3d 377 (3d Cir. 2013)

Corte Suprema

Santa Clara Cnty. v. S. Pac. R. Co., 118 U.S. 394 (1886)

United States v. Ballard, 322 U.S. 78 (1944)

Braunfeld v. Brown, 366 U.S. 599 (1961)

United States v. Seeger, 380 U.S. 163 (1965)

First Nat. Bank of Boston v. Bellotti, 435 U.S. 765 (1978)

NLRB v. Catholic Bishop, 440 U.S. 490 (1979) 
United States v. Lee, 455 U.S. 252 (1982)

Corporation of Presiding Bishop V. Amos, 483 U.S. 327 (1987)

Employment Division, Department of Human Resources of Oregon vs. Smith, 494 U.S. 872 (1990)

Citizens United v. Fed. Election Comm'n, 558 U.S. 310 (2010)

Hosanna-Tabor Evangelical Lutheran Church \& Sch. v. E.E.O.C., 565 U.S. _ (2012)

Burwell v. Hobby Lobby, 573 U.S. _ (2014)

\section{OTRAS FUENTES}

Discurso presidencial 21 de mayo de 2014, disponible en http://www.21demayo.gob.cl/ pdf/2014_discurso-21-mayo.pdf

Diario El Mercurio, 30 de octubre de 2007; El Mercurio, entre el 16 de julio y el 15 de agosto de 2014

Black's Law Dictionary

Federal Register, vol. $79 \mathrm{~N}^{\circ}$ 166, 27 de agosto de 2014 
